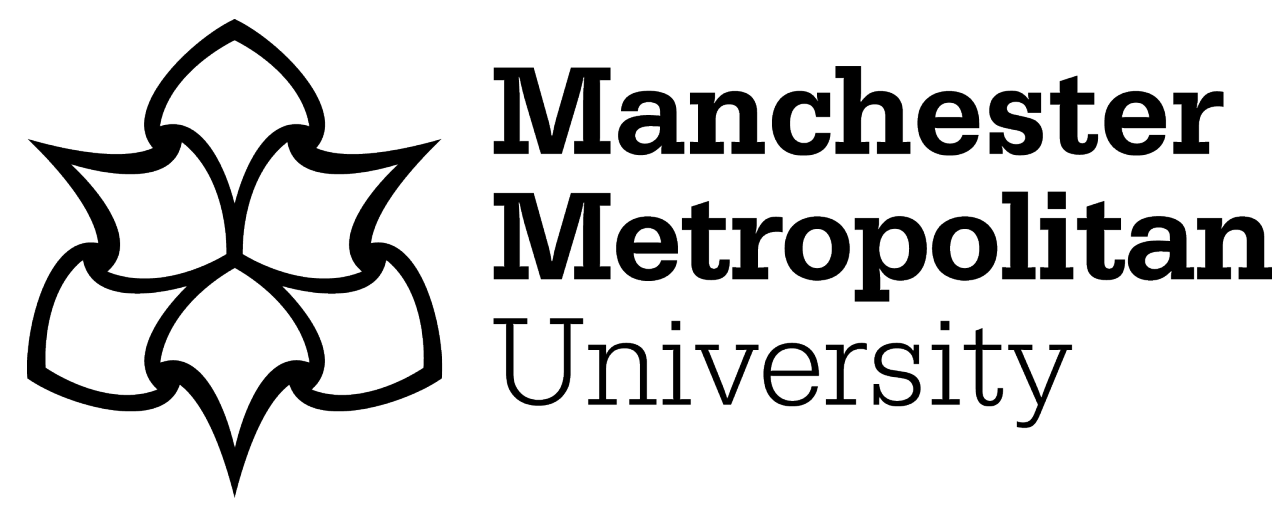

Bell, Barbara and Daniels, John (2019) Sport development in challenging times: leverage of sports events for legacy in disadvantaged communities. Managing Sport and Leisure, 23 (4-6). pp. 369-390. ISSN 2375-0472

Downloaded from: https://e-space.mmu.ac.uk/622504/

Version: Accepted Version

Publisher: Taylor \& Francis

DOI: https://doi.org/10.1080/23750472.2018.1563497

Please cite the published version 


\section{Sport development in challenging times: leverage of sport events for legacy in disadvantaged communities}

\section{Abstract}

This research project focused on legacy around the 2016 BMX World SuperCross event held in Manchester at the National Cycling Centre. In the current social, political and economic climate, the consideration of wider impacts of major events have come under increasing scrutiny. There has been an increasingly critical debate about social benefits, sporting and community impacts, methods to achieve increases in sport participation and event legacy. This paper considers the impacts on people, processes and practice, or 'soft legacy' of the event, through the realistic evaluation of two BMX projects which were based on hosting of the BMX World Cup event. The impact of attempts to leverage social and sport development impacts, in particularly challenging circumstances and communities are highlighted, applying a Realistic Evaluation framework (Pawson and Tilley, 1997) on two programmes. Results showed that though the programme of Street BMX was successful in reaching over 500 participant as planned, there was no evidence of transition into BMX community track sessions. In the targeted event-based programme there were positive benefits to the participants, but limited impact on their educational outcomes. This paper highlights the implications for those planning event-based sport development interventions attempting to engage hard-pressed communities.

Keywords - soft legacy, community sport, BMX, major events 
Sport development in challenging times: leverage of sport events for legacy in disadvantaged communities

\section{Introduction}

The emergence of a growing body of literature around events, legacy and impacts beyond sport has been one of the key features of the 'golden decade' of sport in the UK, announced by Andy Burnham, as the then Secretary of State for the DCMS in 2009 (BBC, 2009) and reinforced in the post 2012 era in UK Sport policy (DCMS, 2012a, b). The expected impacts both to hosting communities and the nation, of economic, social and sport participation benefits were highlighted as key to leveraging London 2012 legacy well beyond the Games. Despite the challenging public sector economic environment, and limited evidence of positive impacts on sport participation, Governmental expectations of sport and sporting events are arguably even greater, evidenced through the level of investments in major events since 2012 .

However, expectations and projections around sport events have not been without criticism or debate. Grix and Carmichael (2012) highlighted the political and ideological processes in the leverage of events, based on the notion of a 'virtuous cycle' link to mass

participation. Several authors (Taks, Chalip and Green, 2015; Taks Greeen, Misener and Chalip, 2014; Misener, Taks, Chalip and Green, 2015; Misener and Mason 2009) have also examined the often flawed approach to legacy building in sporting events aimed at the stimulation of sporting or other impacts in communities. The assumptions of the 'trickledown' of 'inspirational' impacts of sporting events has also been widely criticised as often too optimistic and simplistic in the assumed impact on participation and Physical Activity (Weed, Coren, Fiore et al, 2015; 2012). 
Leverage of sporting or other benefits is based on the planned and focused work, strategies and tactics to increase the potential for outcomes beyond the event for hosts and organisers (Chalip, 2006). This is differentiated from the 'legacy' of the event, which is the impact of this leverage approach, whether positive or negative (Preuss, 2007; 2015). Such legacy can be tangible and intangible, or 'hard' or 'soft'. Many studies related to event leverage focus on the hard/economic or infrastructure impacts. However, other studies (Chalip, 2006; Misener and Mason, 2009; Bell and Gallimore 2015; Taks et al; 2014 ) have considered the leverage of 'soft' legacy'; in people, processes and in communities hosting events, or linked to spreading benefits to wider communities.

This paper is based on a small-scale research project undertaken in 2016/17 around the Supercross BMX World Cup event, held for the third time in Manchester at the National Cycling Centre (NCC), early April 2016. It is focused on 2 specific projects, supported by Sport England, provided by the Eastland Leisure Trust [ELT], operators of the NCC to leverage a community sport legacy from the event ${ }^{1}$. The first project was a Street BMX outreach project, to stimulate participation and engagement in BMX. The second project was a targeted intervention using BMX training at the $\mathrm{NCC}$ track, in the lead up to the event, with a group of children attending a 'Pupil Referral Unit'2. Also part of the leverage approach was the provision of events and coaching programmes in Manchester Schools, but this was outside of the remit for the study. The research applied a 'Realistic Evaluation' [RE] model (Pawson and Tilley, 1997) on very limited resources, to provide an independent evaluation of the programme impacts, and develop insight about the mechanisms and processes involved.

The paper firstly examines some of the literature around event legacy and leverage from major events, particularly around 'sport for change' and social impacts. The paper then examines the background to the intervention and the potential for engagement of youth in 
urban cycling, particularly BMX, particularly with the 'hard to reach' young people in this alternative, informal or lifestyle sport, as highlighted by Jeanes, Spaaj Penney et al (2018).

The main body of the paper examines the methods and key findings in the evaluation of the BMX projects. This was a mainly qualitative investigation, though there is also some registration data, to quantify the level of participation in the programmes and the 'reach' into the targeted communities of South Manchester. Thus, we conducted within a RE framework, an investigation into the engagement of young people in the legacy projects, to examine whether the planned 'legacy' was demonstrated.

Finally, there is an attempt to draw some conclusions about event legacy aspirations and the learning from the BMX-based projects, particularly in reaching hard to reach communities, in challenging circumstances and with a non-traditional sport. As noted above, sport has long been considered a vehicle for positive social impacts, but particularly since the origins of 'sport development' in the 1980's (Collins, 2010). As highlighted by Brookes and Wiggan (2009) and Collins and Haudenhuyse (2015), through local policy implementation into urban environments, sport has been seen as a means to tackle social exclusion and address problems of young people in areas of disadvantage. As Gilchrist and Wheaton (2017) have identified there is a real potential for lifestyle or alternative sports to address the needs of young people who might be disengaged or excluded from mainstream sport, or traditional competitive structures and formal settings. Thus in the UK and elsewhere there has been a proliferation of 'street' based projects -working in non-traditional settings and with hard to reach young people, more at risk of or subject to greater social exclusion (Jeanes et al, 2018; Collins and Haudenhause, 2015) and a growth in the area of 'sport for development' (Schulenkorf, 2012). This movement has not without criticism however, and the assumed 'power' of sport to transform those in otherwise very challenging social circumstances has been subject to some fierce debate, particularly about the evidence of impacts and 
measurement of outcomes (Coalter, 2007; 2010; Collins, 2014). In the current austerity climate for public investment, with the inherent inequalities across communities under pressure (Widdop et al, 2018) any public investment into major events for their sporting or other impacts into disadvantaged communities needs careful consideration.

\section{Event legacies and non-mega events: communities and sport development impacts}

Government policy in sport has increasingly supported the notion of sport for development and 'addressing underrepresentation' (DCMS, 2015: 20). Consequently, support for those deemed 'hard to reach' such as women, girls, the disabled and those in low socio-economic groups has appeared high on recent government agendas (DCMS, 2002; DCMS, 2015). Such policies have been criticised for a lack evidence of mechanisms and processes, particularly in tackling persistent social inequalities (Coalter, 2007). More recently, studies are recording positive outcomes to suggest that sport can develop social skills among youth from vulnerable backgrounds (Riley et al. 2016), improve social connectedness and enhance a sense of community (Sherry, 2016). Increasing levels of social participation has also been linked to a better quality of life and a 'hook' back into education (Sherry, 2016). Alongside this trend of expected social impacts from sport, evidence has also been building to suggest that sports events may stimulate a developmental legacy by inspirational impacts on youth sport participation. This has prompted growth in the number of community sports projects linked to national and international sports events (Richards and de Brito, 2013; Taks et al., 2015). However, Chalip (2006) warned that rhetoric concerning legacy outcomes of sports events are generally hoped for and desired rather than planned for and delivered. Clearly, the political rhetoric around the 'golden decade' of British sport (DCMS, 2012a,b) emphasised this in the lead up to and immediately following the London 2012 Games and more recent policy announcements (DCMS, 2015). Sport development policies have demonstrated a commitment to inclusive practices and have typically targeted specific 
populations, often with young people as the focus. Arguably since the very inception of sport development by the Wolfenden Report of 1960 (Bell, 2005; Collins, 2011), youth has been the focus of efforts to improve both the reach and impact of sport. More recently, young people have been the target of 'Olympic legacy' projects based on a virtuous cycle theory (Grix and Carmichael, 2012) that explains increases in sport participation based on the inspiration of Olympic and Paralympic success. However, this assumed logic model of inspirational impacts has been criticised as being incomplete (Bloyce and Smith, 2012; Weed et al , 2015), particularly when looking at the translation into Physical Activity and 'mass participation' related to national statistics for activity and sport engagement.

In theory, the sport of cycling should yield a good return on investment in terms of sport participation and impacts on youth engagement. British Cycling again demonstrating they are world leading, with 12 medals in Rio 2016, including 6 golds, building on London 2012 and previous successes. The city of Manchester has been home to the National Cycling Centre (NCC) from 1994 and since hosting the Commonwealth Games (CWG) in 2002, has been at the heart of the impressive medal factory for British Cycling for over 2 decades. While there were no GB medals for BMX events in Rio, Manchester has a rich history of BMX development, including hosting the UCI BMX Supercross World Cup events in 2013, 2015 and 2016 in the indoor track built in 2011. In South Manchester, an outdoor track at Platt Fields Park, built to national competition standards with a premise to be accessible to all, hosts regular national events as well as the competition and training for the Manchester BMX club, a leading club in national BMX leagues. However, rates of cycling in Manchester do not reflect a 'trickle down' from this infrastructure and event hosting history.

The funding of projects for a legacy from international cycling events is part of investment in the sport at both national and city level, with Sport England supporting developmental programmes through partnership working across City Council, ELT (facility 
operators), schools and British Cycling. However, the partners are also acutely aware of the need to demonstrate programme impact and develop understanding of how their programmes work, hence the development of the research project with the local University.

In the Sport England Active People surveys (2005-2016) participation levels in the city have consistently been below that of regional comparators. Only $4.4 \%$ of the adult population did any cycling 3 times per week, compared with $6.1 \%$ in Liverpool and for those just occasionally cycling (once per month) the figure was $10.7 \%$ compared to $16.6 \%$ in Liverpool (Sport England, 2016). Liverpool has similar socio-economic indicators, but without the elite facilities, performance pathways or similar levels of schools based cycling programmes. Child health and wellbeing measures were also lower in Manchester compared with other regional profiles (Public Health England, 2018). A high proportion of children live in low-income families, and a relatively high proportion (9.4\%) of young people $16-18$ are classed as Not in Education Employment or Training or NEET, indicating the challenging circumstances faced by many young people in the city. Only $11.9 \%$ of children are active at the rate (one hour per day) advised as appropriate by the Chief Medical Officer, compared to the regional average of $13.2 \%$.

BMX has also some issues with engagement and investment. In what has been described as 'appropriation' of this alternative or lifestyle sport into the sport of cycling, there are criticisms of a commodification and 'sportification' process (Edwards and Corte, 2010; Reinhart and Grenfell, 2002). This commodification might work against the growth of participation in young people in more difficult circumstances, due to increased costs and regulation of otherwise 'free' activity in parks and streets (Gilchrist and Wheaton, 2017).

\section{Legacy Plans and SuperCross World Cup}

Essentially the literature above highlights the assumptions underpinning the work of the City Council, that major events can potentially inspire youth to engage in sport for 
various reasons, but rates of activity shown in surveys and national health indicators shows this 'trickle down' is far from clear. Manchester's designation as a 'City of Sport' seems to have had limited impacts on activity or participation attributable to the elite sports based there. Accolades as the leading 'city for sport' in the UK by ESPN (Parker et al, 2015; ESDN, 2015), are mainly based on responses by sports fans to the opportunities for watching rather than playing sport. The City Council have supported over 216 major events hosted in the city since 2002 , resulting in over $£ 92 \mathrm{~m}$ of economic impact and additional investment of $£ 37 \mathrm{~m}$ (Manchester City Council, 2013). Cycling was highlighted as key partner in this success, and one of the cities 'Tier one' sports for investment.

In attempting to leverage the benefits from UCI BMX World Cup events at the NCC, Event organisers had previously incorporated into one of the final evening intervals a schools BMX race. This gave young and novice BMX riders a role in the major event, as a culmination of the schools coaching programme for the city. For 2016 this emphasis was changed, to develop two new programmes to create a 'legacy' from the event. The overarching aim of the evaluation research commissioned around the event in 2016 was to examine the impact of these legacy programmes. Specifically, a key objective was to establish and monitor the number of young people taking part in one or more Street BMX activity sessions and the transition of participants involved in the Street BMX activity to at least one formal session (Urban Expression) on the Platt Fields Park BMX track. The project was designed to investigate the impact of the two strands of the BMX legacy programme on the young people taking part, and analyse participant characteristics of the Street BMX legacy programme. Essentially the work was commissioned by the Eastlands Trust to evaluate the delivery, process and effectiveness of both programmes 
The ELT BMX Supercross Legacy programmes:

1) Street BMX - delivery of fun, taster, outreach sessions of BMX, coach, bikes and equipment provided across community sites across S / E Manchester. Coaches would offer one off sessions to clubs and community groups, in parks or open spaces, or at community events. The aim was to increase participation in BMX either informally (recreational cycling) or formally (eg at Urban Expressions, BMX club, track sessions)

2) Event - based Development programme - A term time, weekly track based programme of training with a small group of local young people, in a pupil referral unit (PRU). This was taking place in the weeks prior to and following the BMX Supercross event. The young people were to train to 'perform' in a race on the track during the event, and follow up with cycling coaching, volunteering or mechanics based training, depending on interest and progress.

\section{Methods and challenges of the BMX programme legacy evaluation}

This section overviews the evaluation methodology, data analysis techniques and ethical stance taken in the research. The evaluation took a multi- and mixed-method case study design (Yin, 2009), combining both qualitative and quantitative data in order to fully capture the process and outcomes of the BMX Legacy Projects. Pawson and Tilley’s (1997) 'Realistic Evaluation' approach, investigating the interactions between the context, mechanisms and outcomes achieved in the programmes, was developed in consultation with ELT during February/March 2016. During the evaluation (April-September), several meetings took place with programme management for the 'theory of change', updating on progresses and to develop understanding of the mechanisms and processes during the programme. A logic model illustrating the 
connection between the programme mechanisms and its outcomes and to guide the evaluation design, was developed for each project, as shown in figures 1 and 2.

\section{[Insert Figure 1 about here]}

Based on the assumed theories of change identified, each of the projects incorporated the perspectives of different stakeholders along with data from registrations provided by the coaches. Resources for extensive monitoring and data collection were limited. All participants were provided with information sheets about the research and individual informed consent for interview, either face to face or via telephone was obtained. All methods and tools were approved via the University ethical approval process prior to data collection.

For the school-based referral group, in loco-parentis consent via the school and lead teacher of the group was obtained, and individual children also consented to interviews at the follow up visit. Meetings with PRU participants took place at the track - during the BMX World cup and 1-1 interviews at their regular base out of school. Regular observations took place at the BMX track outdoors in Platt Fields park of the supervised BMX activity at the Friday night 'Urban Expressions' session. This enabled an in-depth analysis of the interviews and informal conversations with participants, coaches, parents and volunteers involved and also contributed to recommendations about the communications around BMX. Thus, the range of methods used helped build a better understanding of the context of 'BMX' in the formal and informal settings of the track, street or park.

\section{[INSERT Figure 2 about here]}


The framework of 'realistic' evaluation considered the interactions of the contexts, mechanism and possible outcomes achieved on both programmes - but it was also realistic in the sense of being constrained by 'real world' issues of time and resource to complete a thorough and robust evaluation of the impacts at different levels. No economic analysis of costs or benefits was included - partly as this information was considered sensitive by ELT, but also that the cost of the data collection and analysis required to produce any evidence of social return on investment was considered prohibitive. Detailed questionnaires on activity and health indicators were deemed inappropriate for the Street BMX participants. The indicators of the achievement of intended outcomes at the PRU were based on teacher assessment of behavioural changes and educational status (ie back in mainstream school or having achieved a qualification).

[INSERT TABLE 1 about here?]

\section{Process and procedures in the evaluation}

In the Street BMX programme, as indicated in the logic models, outcome measures were based on increases in or indications of PA level through BMX engagement in organised sessions, measured by analysis of names and addresses, matched with postcodes from the details obtained on outreach sessions.

Informal interviews and observations with participants and parents at track sessions and with the coaches and leaders aimed to get their perspective of any changes in the participation in open access or club sessions at Platt Fields track (where many of the outreach sessions were planned). These observations took place in monthly visits from April, to September to the track and 'Street BMX' sessions in community venues. 
Though the planned visits to the track took place, due to low numbers and late changes to arrangements for the community engagement sessions, there were no completed observations on the community 'Street BMX' sessions. Follow up interviews (2) were undertaken with selected youth workers by telephone, to get feedback and indications of follow up actions. These discussions focused on the youth workers perceptions of the impact of the sessions on cycling engagement or attitudes to BMX, and how the sessions related to their wider objectives with the potential for any ongoing activity with the NCC or BMX track sessions.

Though focus groups were originally planned, less formal conversations were undertaken with parents and children at the regular observations at the track. These informal conversations provided useful insight into the attitudes of young people and their parents on the BMX projects. This also helped with the analysis of in-depth interviews with other stakeholders, as it identified the benefits and limitations of the BMX legacy programmes and the potential impact on wellbeing, health and personal development of the young people concerned. As young people did not complete any questionnaires regarding their Physical Activity, limited inference could be made about the health or activity status of the children taking part, except through indirect, qualitative methods. At none of the observed sessions were any children identified that had taken part in a 'Street BMX' session or had been to the Supercross event at the NCC.

\section{Stakeholder in-depth interviews}

In depth, one-to-one interviews were carried out to capture the experiences of a range of stakeholders:

- the BMX legacy programme staff in the design and delivery of the programme

- the volunteers and coaches supporting the sessions and, 
- Youth, education and community workers who had been involved with children attending organised StreetBMX sessions and Urban Expression NCC based Track sessions with PRU pupils.

Interviews with BMX Legacy Programme Staff explored staff working practices and service ethos plus issues concerning partnership working, with Programme Lead and several of the staff involved in delivery. The interviews took place at several intervals throughout the course of the BMX legacy project. Three staff members were interviewed informally on separate occasions to ensure progress and change in working practices was monitored and recorded. A formal interview with the lead coach on the legacy programme who had delivered the work with PRU and also many of the 'Street BMX' sessions took place in July towards the end of the programme and in a follow up interview sixth months later. Various emails were also exchanged with programme staff to check on arrangements and discuss the sessions during programme delivery.

Youth and community staff and teacher interviews over the phone or in person were undertaken with both the street BMX and PRU BMX event programme. An in-depth interview was undertaken with a lead teacher at the PRU in July after completion of the programme. A brief follow-up interview took place by phone in November, to follow up on 6-month outcomes and ongoing engagement with BMX. Due to changing arrangements with the PRU, school and the youth group involved, it was not possible to complete any further interviews in the 12-month follow up, as the schools contract had been terminated.

All formal interviews, with the exception of telephone interviews, were recorded with permission and informed consents obtained. Transcription of the interviews enabled thematic analysis (Braun and Clarke 2006) to take place. Field notes and observations from visits also assisted with interpretation. Detailed notes and memos from observations or telephone interviews were also coded then thematically analysed. The process of analysis involved various stages of reading and coding relevant sections of text, then grouping and sorting both 
inductively and deductively, into themes related to the Logic model, context, mechanism or outcome patterns. The sections below provide a narrative of the findings and interpretation from across all the qualitative data and include some selected quotes to illustrate the key themes identified.

The follow up, 1-1 Interviews with PRU pupils took place in July, towards the end of the school year, along with the leader of the unit, who had worked closely with the group. Unfortunately again a consequence of 'real world' messiness in the evaluation process (Daniels, 2018), these were deferred due to GCSE exams for the Yr 11 pupils involved and by then all of the (3) participants in the Supercross event had left the unit, for a variety of reasons not related to their BMX project engagement. Even though at least one had achieved their outcome measure of completing a skills based course, only one of those involved with the event completed the cycle mechanics course with British Cycling at the NCC before the end of the academic year.

\section{From Street to Track? Street BMX Programme impacts and mechanisms}

\section{Mechanisms in Street BMX}

In the Street BMX, coaching was focused on giving the young people an introduction into the skills related to BMX riding, performing tricks and the potential to improve technique, safety, bike handling and therefore build confidence. The coach introduced activities like mini-races or skills competitions to maintain interest and add some elements that got young people more engaged. Based on the interviews with programme staff, the coaching was very successful, but there were concerns that in just one or possibly two sessions it was difficult to build up a rapport with the participants that might encourage young people to turn up independently to the urban expression or open track sessions at weekends. 
In the main these were one-off interactions as evidenced by the limited registers taken at the events. Based on the initial interviews and confirmed in the 12 month follow up, the lead coaches' confidence had grown over the course of the programme, so they were satisfied that the sessions provided a positive, active and engaging session - a point reinforced by the youth workers and leaders they worked with in the interviews notes above.

However, simply organising and planning the sessions in parks and with youth groups had been a considerable and unanticipated challenge. There were complications with staff at the centres, other Manchester City Council (MCC) staff regarding permissions to operate in parks and the weather. This meant that many sessions were planned and then changed, cancelled or had to re-locate at short notice. This unanticipated challenge was reflected in the interviews and interim reports by the programme manager. The sessions were subsequently targeted to existing community groups, rather than the original proposed approach to parks and open spaces, in a 'pop up' session, an important contextual factor in their success.

Social media (Instagram and Facebook) had been incorporated into the efforts by the coaches to promote the sessions and show images of the BMX programmes, but there had not been any analysis of the impact of this. The interviews and informal discussions with the coaching team also reflected some frustration with the timing and form of marketing support the programme had. Despite this, the registers and feedback from community organisations showed that the Street BMX did engage children over 500 young people from across South Manchester, mainly focused on areas of deprivation, as discussed in the section below. The aims, in terms of the nature of the sessions provided and the target population, were clearly met. This appeared to be a result of the approach to target identified community groups and the use of community spaces close to their base.

Perspectives of Youth workers - Street BMX impacts and transitions

Key themes identified from the interviews with Youth workers related to: 
- Nature of the activity (related to enjoyment / thrills/ novelty)

- Territoriality (in and out of area working)

- Group cohesion -( benefits of, tackling as a group, co-operation and competition)

- Gender - benefits to confidence for girls in girls only sessions

- Staff and resources - (barriers to ongoing engagement with Platt Fields park sessions, limits and constraints, safe spaces to enjoy)

There were clearly positive evaluations of the Street BMX sessions by the youth workers. Once children had the chance to get onto bikes they were active, engaged fully and enthusiastic about their experiences afterwards. BMX appealed to both boys and girls in the mixed sessions but girls in particular welcomed their 'girls only' sessions, particularly when they felt they were in a little more of a private area, even if this was a car park or open space by the community centre/park.

Both Youth workers commented on the way in which the coach managed to get young people working together. Girls were more likely to need more encouragement to visit again but all groups would have more sessions of BMX at their club - not necessarily every week or formally for a set programme. The programmes of many existing youth groups were designed to include a range of activities but BMX was seen as part of wider programme. Taking the children to the track at Platt Fields (even for those who were relatively close) was unlikely or difficult to do, because of staffing - too few staff to spare someone to take a small group to the track; or due to concerns about working 'out of their area'.

The issue of territoriality for children and young people - getting to the NCC or Platt Fields was difficult from their club - travelling across the wrong postcodes. Either their parents would lack the resources (car) to take them, or the time/enthusiasm to make the journey, or children had concerns about safety in the parks or areas outside of their centres. ${ }^{3}$ 
Youth Staff were confident that all their children who had taken part could ride a bike, most had their own, though it was more likely they were into mountain bike type riding (not on organised trails but around local streets/open spaces). They considered the sessions would have contributed to more confidence and therefore more likely to do more cycling - but with dark evenings, they were unlikely to be doing this down at the track. There was no real assessment of the activity by children outside of their sessions but overall the youth workers felt this could be part of their overall programme in future, given sufficient staffing and some support for transporting children to the track.

The Urban Expression timing (6-8pm) on Fridays or Saturday evening at the NCC was seen to be an issue with several groups. This was in direct competition with their general youth sessions in the clubs - ie they could not spare people to take small groups and the whole group would only want to do BMX cycling occasionally unless it was on site.

\section{'Better than hanging around' The views of parents and young people at Urban}

\section{Epression sessions}

Parents attending the Urban Expression were generally accompanying young people under 12 or small groups including some family groups. Many were mothers who were keen to express their approval of the format and availability of the sessions on the track. Several parents who had been involved with schools-based BMX sessions brought their children along to other track-based sessions, including the Friday night Urban Expression sessions. In all of the visits insufficient numbers of young people were identified for any of the planned group-based discussions. Small numbers were attending, many of the children were reluctant to talk or they were perceived to be too young to approach without parental consent, or simply too busy riding the track or hanging about with their friends.

Other parents had brought children to the park, without really understanding what the sessions were about. Often these were in large family groups with children taking part in a 
range of activities in the park (basketball, skateboarding, games of cricket, football, bike or scooter riding). Parents were enthusiastic to give the children the opportunity to ride the track for only $£ 1.50$ including the bike and all equipment. None of the parents spoken to had been aware of the sessions through any marketing, website or knew of any links to the Supercross event. They often lived relatively close to the park, having walked or taken a bus on evenings with good weather. Poor weather meant very few people in the park and very few riders on the track.

One mother who had brought her own 11 year old and several friends on the bus, was making her first visit to the track, though her son had been before with school. She had plans to take the boys to the indoor track - provided it was going to be as cheap. Her main reason was to give her son a good experience of riding his bike which he did a lot of at home, but also to learn how to be safer on it. She talked about how the BMX session of 2-hours 'really tired him out' all he did when he got back was go to bed and sleep - and how it 'kept him from mischief'. This was the sort of activity she preferred him to do i.e. better than just “...hanging around or going off with friends." This was something she said she would like to do more of and now she had seen the track, she would look out for details of when they could come as a family. Parents seemed to appreciate the emphasis on safety and use of protective equipment.

Most of the young people of 11 years or above seemed to come unaccompanied, or if younger, their parents were elsewhere in the park and just paid/booked the children in, then watched at a discrete distance. The lack of signage and clear instructions about what to do and what was happening at the sessions meant that some parents or children were unsure if sessions were open or not, or what the arrangements were, often hanging about at the gate observing before coming in. Volunteers were regularly sent out into the park to give out flyers to encourage people in the park to bring their children. 
Programme Staff and coaches '/volunteers' perspectives of the impact of Street BMX on regular BMX sessions

Based on the figures provided by ELT, there had been lower engagement in the UE summer sessions in 2016, compared to the previous year, when no additional legacy programme had taken place. Several weeks recorded only single figures of participants, particularly in the poor weather of April and May. Consequently, informal interviews with the staff coaching on Fridays focused on their view of the possible transitions from street style BMX engagement to the track sessions. The final interviews were conducted to reflect on the summers activity on the track. At the final session attended in early September, there were no participants, with poor weather and the park in darkness by $7.00 \mathrm{pm}$.

The programme staff and volunteers focused on their views on the reasons for poor numbers: they referred to a lack of support for marketing, problems with track signage and concerns of accessibility. Staff were clearly very committed to growing the sport, but felt some constraints at the track and its management. From early on in the summer, it was clear that the achievement of the stated KPIs in transitions to UE was going to be a challenge for the Street BMX, and it quickly became clear hitting the targets was extremely unlikely. On every visit before July the research team were informed that the sessions would be much busier in the school holidays. However, this expected upturn never occurred. Also, with the delays and gaps in the Street BMX sessions, it was clear that any progression into regular BMX was unlikely to be picked up at the track. It was not possible, without clearer tracking of individuals, to do follow up investigations into the level of cycling or general activity as a consequence of taking part in one-off Street BMX sessions. The coaches certainly did not see this process working, except through schools visits, where they recognised some children as coming from their schools sessions.

The coaches and volunteers expressed concerns about the lack of clear marketing and promotion and general information about BMX and cycling at the track. Coaches felt under 
pressure to not only maintain a fun and safe session, but also to act as a recruiter for the session by inviting people in from the park. This suggest that such community programmes require clear roles to allow for effective delivery of programme mechanism if outcomes are to be achieved. Volunteer Mike (pseudonym), when asked if he went to the BMX club sessions responded:

“...no, not really my thing, I prefer to come to this' - the club was seen as about serious 'riders' and 'racers'... this Friday night is all about 'chilling and just relaxing on the bike', 'hanging out with mates."

An important aspect of the 'context' was that the 'closed' nature of the BMX track was seen as potentially off putting for informal cycling. Young people were likely to be using the track unofficially when it suited them, and had to be discouraged from riding with their own bikes often without brakes and no safety equipment (e.g. helmets/gloves). On my final visit, John (Coach) stated:

“I've had to just chuck 4 lads off....they wouldn't stay and ride on our bikes with helmets so they had to go...." (Field Note: no other young people were out on the track that evening)

The coaches also pointed out that the reduced programme for the track meant that young people actually had very few sessions they could access by late September, so if any of the children attending Street BMX were interested in taking part, they might be going to open sessions at weekend. As far as they were aware, no one was going to club sessions having been in the Street Session, but they couldn't be sure. Overall, the coaches based at the track were concerned that young people were not aware of the sessions available to them, and the NCC website was not very helpful in promoting to young people or their parents.

This aspect of the evaluation therefore highlighted that the funding enabled an extensive programme of Street BMX sessions across the area of South Manchester, including 
in some of the most challenging communities for activity. There was some evidence that the Street BMX coaching and events might have raised awareness of BMX through positive experiences in sessions in clubs, groups and events. There was evidence of the successful and positive approach in the less formal Street BMX sessions provided. Despite this engagement in extensive sessions, there was no clear evidence of transitions into regular Urban Expression or other BMX activity from those who participated in the Street Sessions or taster events - ie a failure in the logic model proposed, and no achievement of the planned KPI measures in transition to regular BMX sessions.

\section{Progression in Street BMX and the achievement of the legacy objectives}

Quantitative data on engagement was recorded at a number of Street BMX activities/ venues, via registers completed by NCC staff. These included: Indoor track at NNC Sessions, Parks, church and youth groups and schools sessions. Registers recorded 285 participants across 10 events and sessions. These indicted in summary:

- A significant proportion of female participants, important given the current efforts to make sport more accessible for women and girls.

- A significant proportion of BME children reported by coaches

- A purposeful reach in terms of socio-demographic area, based on postcodes (high BME populations).

A further 298 participants were recorded at various community and education events such as 'Schools Together' days and activities at local voluntary organisations or community events though individual registration data was not taken. Thus, 583 participants engaged with the project representing $97 \%$ of the agreed target of 600 participants.

The registrations for these sessions by gender (where recorded) can be found in Figure 3 and clearly indicated the NCC, Schools Together and the 'BiggaFish' group from 
Trinity House as the most popular sessions in terms of participation. The NCC and the Ladybarn Community Centre sessions were particularly successful in engaging females for the mixed sessions. The participants also reflected the ethnic diversity of the areas targeted, though details of ethnicity were not captured on registrations.

[INSERT fig 3 here: attendance at Street BMX sessions]

The 'reach' of the project into local communities

Of the 285 participants, 99 recorded post codes were obtained. Postcodes were concentrated around the Southeast of Manchester, including Hulme, Withington, Burnage, Rusholme and Levenshulme. These districts are acknowledged as some of Manchester's most deprived areas according to the 2015 Indices of Deprivation published by Manchester Council (2015). According to Sport England, it is in these areas that participants are 'hardest to reach' or engage in sport (Sport England, 2015. These are also the areas hit hard with austerity climate issues.who suffer from low participation rates in PA in general, and organised sports in particular (Public Health England, 2018). Given the locations of the Street BMX sessions, reflected in the postcodes of participants, the programmes successfully reached the populations of young people in the areas around the NCC and Platt Fields Park and made some contribution to the sporting capital of young people there, albeit over a short engagement period.

\section{From Event to Engagement? the impacts of BMX event-based programme with PRU based young people}

A BBC ' Get Inspired' film made with the PRU group at their training event, suggests that the sport of BMX has potential to have an impact on children who are struggling with mainstream school, who have significant issues to deal with and who may be at risk of permanent exclusion. 
There were clear disciplinary, behavioural and physical challenges involved in the intensive training at the track, which were recognised by the coach as presenting a clear challenge to him also: "it was by far the hardest thing I've done" - BMX Coach of the PRU group

\section{The impact of the Event at the SuperCross World Cup}

The first author met the children, coach and teacher at the 2016 UCI BMX SuperCross World Cup event in April, at the culmination of their training, where the young people 'competed' in a race during the interval of the elite event. They were kitted out in specially designed shirts (designed by one of the group riding) in honour of their successful completion of the programme. Unfortunately, only 3 out of 14 young people who had gone through the training took part, despite the clear enthusiasm. As the event was in the Easter holidays, several were on holiday with families or simply failed to turn up at the collection point, with one pupil injured on the track in the final training session and unable to take part. It was clear that this small group were nervous and found this experience to be a great challenge, despite the excellent and calming support from their coach and teacher. However, it was also clear that the successful completion of the race and the rest of the experience surrounding the event was seen as quite an achievement and something they had pride in completing.

This was quite clearly expressed in the BBC 'Get Inspired' clip (BBC 2016, see link below $)^{1}-$ where the only female taking part, expressed her views on how she was looking forward to the event. At the event they were supported by volunteers from the BMX club, and accompanied round the track. However it was clear, in front of a large and vociferous crowd of real BMX enthusiasts, this was a significant challenge to the young people.

\footnotetext{
${ }^{1}$ http://www.bbc.co.uk/sport/get-inspired/35947269 - Film by BBC of the group training (8 April
} 2016) 
Despite the great experience for the 3 riders, non-attendance by other members of the group was noted as a key disappointment, particularly given the extra time put in by coach, volunteers and the accompanying teacher. Unfortunately, by July when the follow up visit took place, none of the 3 young people who had taken part in the event were still attending the PRU, so no first-hand accounts of their reflections on this experience could be obtained. The reasons for their problems in mainstream education had not been addressed despite their successful engagement with the BMX project. The teacher concerned was careful to point out that their behaviour while at the unit had definitely improved during and after the BMX programme, and their ongoing personal issues were external to their positive improvements whilst at the unit and taking part with BMX and other activities.

Other students who took part in the pre-event training and some subsequent sessions at the track, (including the mechanics course) were no longer at the unit having returned to mainstream school. This could be seen as a positive outcome, related to the indicators for the legacy programme, but clearly the BMX sessions alone could not account for the relevant educational outcomes achieved by the young people, something their teacher/youth worker had worked intensively on an individual basis with young people involved for many months.

\section{Young participants perspectives on their BMX experience}

There were 4 young people still attending the unit in July who agreed to be involved in short interviews to discuss their experience and the value of their BMX programme. They had gone to most if not all of the sessions available and were all very positive about the sessions. Some expressed regret at not having taken part in the event. Either due to problems with being 'sent back' to school in the week before end of term (thus missing final training session), or the event being in the Easter break, they had simply 'forgot' about it. All the young people interviewed referred to the importance of their enjoyment of riding the track for achieving something positive from the sessions. 
After analysis, the following themes were identified in the pupil interviews:

- Enjoyment of BMX

- Personal development and growth - skills and confidence

- Impacts on future and or school

- Role of the coach

All the pupils highlighted fun, enjoyment and excitement of the sessions on the track as an important contextual element of the programme:

\section{"It's just more fun to do, you just learn different things you learn something new every day. ” Pupill \\ "I wanted to do it because it sounded fun-and it was good." Pupil 2}

The benefits of the BMX based programme at the track to personal development and growth was clearly highlighted in the interviews. They identified important contexts such as a positive coaching environment, which was clearly important to the children, it facilitated significant outcomes, interacting as a group, talking and supporting each other, they were treated with respect and had to listen (for safety and to learn new skills).

\section{"It just like gets us away from like doing bad things and gets us all interacting with each other." Pupil 3}

BMX riding wasn't seen as a serious activity, but it was something new and different, something they could do with their mates - but not get into trouble doing (ie it kept them out of trouble):

\footnotetext{
“It's just like because you can interact more with people and it's not as its serious but not as serious as the others. So yeah, its good fun, but at the
} 
same time you're having fun with your mates, so like, you're not about on the streets getting into trouble and stuff." (Pupil 3)

It was useful to be trained to ride properly, children appreciated the opportunity to get these skills in riding such as learning new tricks, going faster on their bikes, experiencing the track and learning how to look after their bikes. This reinforced how young people perceived developing new competencies as contributing to their sense of achievement.

\section{"They taught us like different ways and like, how to do tricks and stuff and how to move quicker on the track." (Pupil 3).}

The experiences itself was both fun yet challenging (scary/exciting/fun) but also about learning (skills, how to get on, being more safe/ more independent / save money (on bike repairs):

"It's good they teach you because, like it could save you money and stuff."

$$
\text { (Pupil 3) }
$$

Children referred to how this course was going to help them - seen as a diversion from trouble, and an opportunity to learn new skills with possible career benefits, vocational training routes, or getting them back into school. They noted it had improved their attitude:

\section{"Because with good behaviour and stuff and like interacting with things...I used to have an attitude...I could go back to school." (Pupil 3)}

And having taken part in the whole programme (despite missing the event), something they were proud to have completed. Asked about how he felt about going on the track:

\section{"It was scary at first, but then I did it...I still got a lot out of it." (Pupil 1)}


Some children were clearly engaged with other sports out of school - all had the ability to ride a bike and were active outdoors. They indicated that BMX was better than some more traditional sporting experiences (eg with Rugby) which seemed to have put them off from engaging in activity - as it wasn't all about being in a team or competition - they enjoyed being faster and competing, learning new things together as a group and were happy to work together. The teacher of the unit reinforced that the work on the track clearly complemented their approach to using purposeful activity to develop concentration, improve attitudes to learning and experience new physical skills:

\section{"I was really impressed with the whole thing, the fact that health and} safety, the way they took to it, the fact there was a team spirit within it, the skills that were involved and the behaviour on the track."

\section{(Teacher at Pupil Referral unit)}

The teacher's perspective echoed those of the children regarding the challenges in BMX. They enjoyed the sessions so much he was able to incentivise good behaviour with the potential of attending or stopping the sessions if they misbehaved. The positive management of the sessions by the coach clearly reinforced his approaches - a positive mechanism of the programme. There were clear impacts on the behaviour of the group as they got to know the coach and were more confident and respectful of him. From the teacher's perspective, BMX and cycling centred approaches had good vocational and practical potential for this group of boys (and the one girl who had taken part) over and above the physical aspect. Learning about bike maintenance was clearly linked to the aspirations for jobs in engineering or other practical aspects, such as working at sport facilities.

The teacher, experienced in this type of work, considered the engaging, active sessions kept the interest of this notoriously difficult group - and reinforced teamwork, better 
interactions between the group and with the leaders, which would stand them in good stead on return to school.

"These kids have got a problem with authority and following instructions.

We've been there an hour two hours, and Rob's really retained their enthusiasm and interest." (Teacher Pupil Unit)

In conclusion, and after the follow up interview in particular, he reinforced he was still taking other children and some from this first group back to the NCC for more sessions, in order to build on this work. The only aspect he might have improved would be to have a much clearer exit route into mechanics or work at the NCC clearly set out at the start. It wasn't really necessary, from his perspective, to be part of the big event to achieve the most benefits for the young people. If the event had taken place in term time rather than in the Easter break, it would have been easier manage attendance.

\section{Working with more challenging groups- the coaches perspective}

The style of this work was very different to that the coach had experienced before. Though he had some training in dealing with challenging behaviour, the work with the PRU was the first prolonged engagement with anyone other than the BMX club participants or enthusiasts at the track. There were clear developmental gains for the coach as well as the children in a possibly an unintended consequence of this and the Street-based work.

In this session, the key contextual issues from the coach's perspective were to do with the importance of building relationships with the young people over time, to establish their trust and also gain their respect. He was able to gain some understanding of their difficulties and rather complex lives and see things from their point of view - something that the teacher working with them had more experience with. Clearly that only 3 of the group took part in the event was a disappointment. However, all made significant progress in their BMX work, 
and the progression onto bike mechanics was seen as clear 'added value', as was the ongoing relationship with the PRU and the potential for new groups to go the NCC.

Another unforeseen outcome related to the event was highlighted in the engagement of young volunteers from the BMX club (who he normally coached) who were able to gain experience of leadership and support to the inexperienced riders, and also the background working of the event itself. This helped to deliver some 'legacy' in terms of their ongoing qualification as event volunteers with BMX youth ambassador programme.

Therefore, while the programme of BMX training, event and post event engagement was followed as planned (eventually), this was not without its problems. Those children who engaged fully with event and training had positive experiences and showed improvements in behaviour back at the unit. However, very few impacts were solely due to this programme and even so, no clear evidence of any improved educational outcomes was obtained. The one pupil who had gone on to college was arguably positively influenced by the individual level of attention afforded in the programme and support he had received as a result of this engagement with additional training in cycle maintenance. BMX cycling had retained his interest and enthusiasm and provided an interesting programme of activity for him, helping to keep him engaged with the unit, back to school and linked to his vocational aspirations, successfully get a place at a college.

\section{Mechanisms, contexts and outcomes: the leverage process in the BMX programmes}

Though KPIs might have been missed in both strands, there was clear and positive learning at both individual and organisational level, to show how the leverage of a BMX programme was able to contribute to the sporting capital of Manchester and achieve worthwhile outcomes for participants involved. The different contexts in which the programme mechanisms operated clearly had some different impacts on outcomes achieved. The Coaches, Leaders/volunteers /Teachers/Youth workers - all clearly gained in knowledge and experience through this 
programme, contributing to the sporting infrastructure and personnel involved in BMX/Cycling - a perhaps unforeseen outcome in 'soft legacy'. For young people - there were clear benefits to behaviour and attitude for example, in the PRU group children who took part, even if they did not attend or race at the BMX SuperCross event. In the Street BMX programme, delivered across appropriate communities in Manchester, to a good range and number of young people - achieved the planned impact through a mixture of community events and targeted sessions, rather than the original planned context of 'pop-up' delivery. BMX sessions clearly reached the targeted areas and populations and provided positive and engaging experiences for those who took part. The outreach work contributed to the overall programmes of established youth groups, providing important contexts for the programme mechanisms to work more effectively.

Though the awareness of the BMX offer in Platt Fields and NCC was raised, due to territorial or other issues for young people the regular organised sessions (where registers were made available) could not show any transition by participants. Any ongoing participation or engagement by young people was likely to be of very informal or related to ongoing streetbased activity in cycling, rather than BMX specifically, hence almost impossible to verify.

\section{Process outcomes and consequences-reaching the 'hard to reach' with BMX}

Despite the lack of progression for some children with very positive experiences at the track this wasn't necessarily a reflection of a lack of success of the programme. Their complex lives and wider influences on behaviour and progression intervened and their improved behaviour did not necessarily result in longer-term changes the programme was aiming for. However, there was some evidence of the potential for a BMX intervention with 'hard to engage' pupils, with the coaches working in an appropriate way, in small groups under supervision from their regular unit leader, or specialised worker. 
This programme seemed to work best where there was a good match with the ethos of the unit and staff involved. Based on the follow up reflections with staff at the track and unit, the targeted sessions would not need a major event involvement, except perhaps for celebration of completion, or with tickets to watch the event as incentive. A longer programme of work, over a full term rather than 6 weeks, a planned exit route into mechanics and or other vocational training, or other work-related experience at the NCC could potentially produce the outcomes sought.

The research processes thus developed some insight into the relationships in the BMX projects, though there appeared to be some lack of clear 'process' as expected in the logic model. There was some evidence of what Rossi, Freeman and Lipsey (2003) suggest is 'process failure' - ie the programme was not delivered or received as originally intended. Consequently this limited any possible impacts and subsequent outcomes - there was insufficient engagement in the taster sessions provided to do much more than provide a oneoff experience, only small number of the referral group actually took part in the full process of pre- event and follow up training.

Youth groups were reluctant to re-locate their own work to the NCC or BMX track as it was felt to be 'out of their area'. The planned outcomes were arguably too ambitious and or difficult to measure to provide any real evidence of impact - some 'theory failure' therefore contributed to lack of evidence and impact, an issue recognised by the ELT programme staff later. Despite this, the main coach involved clearly developed new skills and competencies in relation to the non-traditional settings of the youth projects or community spaces and halls, and working with the 'hard to engage', which was perhaps an unintended but important part of the 'soft legacy' of the event-funded programmes.

There were other unintended consequences other legacies, for example, the involvement of the volunteers from the BMX club, the increased engagement of youth groups 
and youth workers in South Manchester and the clear ethnic diversity represented in community engagement sessions. The BMX club and their participants represent a range of Manchester and beyond postcodes, reflecting its status as a major hub for the sport, attracting participants from a wide catchment. In contrast, the youth groups came from very targeted areas of South Manchester and despite the low numbers in Friday night sessions, the diversity and inclusion of different ethnic groups was clearly observed.

Therefore the Street BMX programmes have helped to raise the awareness of the sport in local communities, even if the formal sessions were not well supported. Through their engagement with the event in April, the BMX club young members supporting the novice riders had been able to develop their leadership skills and through engagement with the event, had gained significantly in their own development, as this had contributed to their leadership and event training in BMX.

The lead coach highlighted that the coaching he had done on the Street and PRUbased sessions was a real challenge and clearly developmental for him, as he was supported by a manager with some genuine empathy and understanding of the challenges an important context or condition for success. There was clearly an impact on his confidence and the recognition that he was now much better equipped to do this work in the future.

\section{Learning about legacy, leverage and impacts from BMX projects}

There are clearly inherent challenges for Sport Development from major events particularly in challenging environments and difficult groups -as this albeit limited study shows. For robust research 'evidence' this project lacks definitive before and after measures on individuals or groups, controls, for observable 'changes' attributable to the event. The study is reliant on qualitative/observational data, with problems of missing data in registrations and details of participants at Street sessions, a lack of access to participants and longitudinal outcome tracking. There were also practical and logistical difficulties with the 
time frame of the study, intervening school holidays and even the weather. However, this project has clearly illuminated some problematic aspects of event-led sport development research, particularly working with disadvantaged communities or difficult to reach groups, in the messy reality of community sport. Presumed or planned for outcomes remain elusive and perhaps overstated in the original logic model and plans. The original research design was based on assumptions, which proved to be inappropriate, overoptimistic or inaccurate.

Through the qualitative approach within the Realistic Evaluation framework, it was possible to identify the interactions of mechanisms (M) and contexts (C) through interviews and observations. The outcome $(\mathrm{O})$ of positive impact of short term engagements on the competency of young people (O), was achieved through the skills and fun emphasis of sessions (M), in keeping with freedom and self-expression of the informal activity (C), rather than on a competition focus. It was also possible to determine, through the interviews with practitioners and managers, a 'soft legacy' in changes in approaches and in skills related to practice with targeted groups and the so called 'hard to reach' groups. This identified the need for close collaborations with relevant groups and agencies already working with the communities, for example other charities and established PRU units, which provided the important context (C) for the mechanisms of good coaching to be effective.

\section{Building a Legacy for BMX?}

Suggestions for action by ELT and Cycling programme management included the need to establish some ongoing/ additional engagement with research and evaluation of impacts on young people in PRU settings, looking at the link from BMX or other cycling activity and educational benefits in more depth. This 'Realistic Evaluation' of the event-based PRUbased approach was too limited to be able to draw specific conclusions, based on the relatively small group who completed the relevant training. Clearly there is potential for more research into educational impacts on young people, using more refined before and after 
measures of selected attitudes or behaviours, and the impacts on physical activity levels of participants, with more focused evaluations, including more outcome tracking. However, this was beyond the scope of this limited project and the resources available. The evaluation approach had demonstrated the potential for BMX-based training programme of sessions to specialised units, but not fully evidenced the impacts.

To enhance the potential impacts of Street BMX, there was a clear need to develop more effective marketing and promotional material for distribution at Street or Outreach Sessions to established youth groups. Also, to monitor impacts on future users of the track and in informal BMX through more individualised tracking via a form of 'passport' for riders to flag (through the database of registered users) any later engagement with NCC or ET facilities. This required closer liaison with established youth groups, the existing Cycle Hub at Platt Fields Park and other potential stakeholders, for example the local Universities, for increased access to the open track when not in use by clubs.

It was also possible to see the potential for BMX work to link with other related projects in the city, and in the development of more 'open access' tracks across Greater Manchester ${ }^{4}$. Without some appreciation of the different motivations and perceptions of the sport of BMX from the local communities, any similar project was likely to struggle to appeal to those young people who prefer the 'chilling' and hanging out with friends to the regulated, commodified and competitive sport of BMX.

Throughout the whole process, it was clear that the event, the BMXSuperCross World Cup, had a very limited impact into local communities, unless they were already engaged with the sport, a criticism highlighted in many studies of events above (Misener and Mason, 2009; Taks et al, 2014; Misener et al, 2105). There was clear tension in the perception of the sport of BMX, compared to the 'street' activity - based on the strictly regulated access to the track, as shown in previous research into the development of the sport away from its informal 
roots (Reinhart and Grenfell, 2002). There were still unresolved issues regarding the 'open' track sessions and the level of access to local young people, in the management and operation of Platt Fields Park track.

Though relatively small scale this study has demonstrated the complex nature of the legacy debates around sports and city-wide investments. This is particularly topical with the announcement in 2017 of the Commonwealth Games returning to Britain in 2022, following Glasgow 2014 and Manchester in 2002. The assumption of achieving positive outcomes for local communities around major events remains a powerful political argument, even if evidence of actual benefits is difficult to find. The communities in and around our major cities provide significant challenges for sport development - both in ensuring that the communities benefit from these events, and also that inclusive and accessible services can be sustained in the longer term.

\section{Acknowledgements}

The authors acknowledge the support of the ELT Cycling Programmes Manager and staff on the projects for their co-operation and contribution to the research project. The research was conducted on behalf of the Eastlands Leisure Trust, funded with the support of Sport England. Our findings and conclusions are independent, though we have drawn upon reports submitted to ELT and by ELT to Sport England. No individuals are identified in the paper, unless with official title/role and their prior consent.

Notes

1. Eastlands Leisure Trust was established in 2015 , as the facilities operator of the NCC and other Manchester City Council sports facilities and services.

2. More details of the 2 projects are provided in logic models. The original target was for young people Not in Education or Training [NEET] to be recruited, but this was changed to children in a Pupil Referral Unit [PRU] ie taught apart from mainstream school classes temporarily due to behavioural or educational issues 
3. The BBC filmed one of the training sessions and spoke to several participants posting the film onto the 'Get Inspired' website in the lead up to the April event - this was still available in 2017. Their views in the film were considered a good proxy for individual interviews as a 'baseline' of attitudes before the event.

4. At the time of the research, the issue of gang and related violence in South Manchester was a real and genuine concern.

5. The City of Manchester and other Local Authorities have plans for increasing open access BMX parks across the region, as seen in London after 2012, as identified by representative of Access Sports - a national charity contributing to BMX plans in Manchester, as they had in London. 


\section{References}

Bell, B. (2005). 'Evaluating Programme Impacts: Champion Coaching on Merseyside pathways to opportunities?' In Hylton, K., Flintoff, A., Long, J (Eds.) Evaluating Sport and Active Leisure for Young People Brighton: LSA pp. 75-108

Bell, B., \& Gallimore, K. (2015). Embracing the games? Leverage and legacy of London 2012 Olympics at the sub-regional level by means of strategic partnerships. Leisure Studies, 34(6), 720-741. doi:10.1080/02614367.2014.994553

Bloyce, D., \& Smith, A. (2012). The 'Olympic and Paralympic effect' on public policy: use and misuse. International Journal of Sport Policy and Politics, 4(3), 301-305. doi: $10.1080 / 19406940.2012 .746236$

BBC News (2009) Minister promises Sporting Decade, Jan 12009 Online at: http://news.bbc.co.uk/1/hi/uk/7807169.stm accessed 20 June 2016

Brookes, S., \& Wiggan, J. (2009). Reflecting the Public Value of Sport -- A game of two halves? Public Management Review, 11(4), 401 - 420.

Chalip, L. (2006). Towards Social Leverage of Sport Events. Journal of Sport \& Tourism, 11(2), 109-127. doi: 10.1080/14775080601155126

Coalter, F. (2007). Sports Clubs, Social Capital and Social Regeneration: ill-defined interventions with hard to follow outcomes? Sport in Society: Cultures, Commerce, Media, Politics, 10(4), 537 - 559.

Coalter, F. (2010). The politics of sport-for-development: Limited focus programmes and broad gauge problems? International Review for the Sociology of Sport, 45(3), 295314. doi: $10.1177 / 1012690210366791$

Collins, M. (2011), 'Children's sport in policy contexts', in Stafford, I. (Eds.) Coaching Children in Sport, London: Routledge, pp. 276-277.

Collins, M., \& Haudenhuyse, R. (2015). Social exclusion and austerity policies in England: The role of sports in a new area of social polarisation and inequality? Social Inclusion, 3(3) doi:http://dx.doi.org/10.17645/si.v3i3.5

Daniels, J.E., Bell, B. \& Horrocks, C. (2018) Capturing the realities of sports programmes: systematic 'messiness'?, International Journal of Sport Policy and Politics, 10:4, 779 794, DOI: 10.1080/19406940.2018.1513414

Department for Culture Media and Sport (2002). Game plan: a strategy for delivering government's sport and physical activity objectives. London: Strategy Unit 
Department for Culture Media and Sport (2008). Before during and after: making the most of the 2012 Games on line at:

http://webarchive.nationalarchives.gov.uk/+/http:/www.culture.gov.uk/images/publica tions/2012LegacyActionPlan.pdf

Department for Culture, Media and Sport (2012a). Creating a sporting habit for life. London: DCMS.

Department for Culture, Media and Sport (2012b). Beyond 2012: the London 2012 Legacy story DCMS online at:

https://assets.publishing.service.gov.uk/government/uploads/system/uploads/attachme nt_data/file/77993/DCMS_Beyond_2012_Legacy_Story.pdf

Department for Digital Culture Media and Sport (2015). Sporting future: a new strategy for an active nation. London: DDCMS.

De Knop, P., Wylleman, P., Theeboom, M., De Martelaer, K., Van Hoecke, J., \& Van Heddegem, L. (1999). The Role of Contextual Factors in Youth Participation in Organized Sport. European Physical Education Review, 5(2), 153-168. doi: $10.1177 / 1356336 \times 990052001$

Edwards, B., \& Corte, U. (2010). Commercialization and lifestyle sport: lessons from 20 years of freestyle BMX in 'Pro-Town' USA. Sport in Society, 13(7/8), 1135-1151.

ESPN (2015) Manchester beats London as Top UK sporting city online at: http://www.espn.co.uk/espn/story//id/13368888/manchester-beats-london-top-espnuk-greatest-sporting-cities-2015-study Accessed May 1818

Gilchrist, P., \& Wheaton, B. (2017). The social benefits of informal and lifestyle sports: a research agenda. International Journal of Sport Policy and Politics, 9(1), 1-10. doi: 10.1080/19406940.2017.1293132

Grix, J., and Carmichael, F. (2012) 'Why do governments invest in elite sport? A polemic.' International Journal of Sport Policy and Politics, 4(1), pp. 73-90.

Jeanes, R., Spaaij, R., Penney, D., \& O’Connor, J. (2018). Managing informal sport participation: tensions and opportunities. International Journal of Sport Policy and Politics, 1-17. doi: 10.1080/19406940.2018.1479285

Manchester City Council (2015) Indices of deprivation 2015. Manchester: Chief Executive Department. 
Misener and Mason (2009) Fostering Community Development Through Sporting Events Strategies: An Examination of Urban Regime Perceptions, Journal of Sport Management, 23, pp 770-794

Misener, L., \& Mason, D. S. (2006). Creating community networks: Can sporting events offer meaningful sources of social capital? Managing Leisure, 11(1), 39-56.

Misener, L., Taks, M., Chalip, L., \& Green, C. B. (2015). The elusive 'trickle-down effect' of sports events: assumptions and missed opportunities. Managing Sport and Leisure, 20(2), 135-156.

Parker, A., Sarkar, M. Curran, T., Williams, S. (2015) Greatest Sporting Cities 2015 Research Report. A report prepared for ESPN University of Gloucester, University of Bath.

Pawson, R., \& Tilley, N. (1997). Realistic Evaluation. London: Sage.

Preuss, H. (2007). The conceptualisation and measurement of mega sport event legacies. Journal of Sport \& Tourism, 12(3-4), 207-228.

Preuss, H. (2015). A framework for identifying the legacies of a mega sport event. Leisure Studies, 1-22. doi: 10.1080/02614367.2014.994552

Public Health England (2016) PHE data analysis tools. Public Health England. [Online] [Accessed on $15^{\text {th }}$ October, 2016] https://www.gov.uk/guidance/phe-data-and$\underline{\text { analysis-tools }}$

Public Health England (2018) Physical Activity Profile North West region accessed May 2016 - May 18) https://fingertips.phe.org.uk/physicalactivity\#gid/1938132899/ati/102

Reinhart, R., \& Grenfell, C. (2002). BMX Spaces: Children's Grass Roots' Courses and Corporate Sponsored Tracks. Sociology of Sport Journal, 19, 302-314.

Richards, G., and de Brito, M. P. (2013) The future of events as a social phenomenon: exploring the social impact of events. London: Routledge.

Rossi, P. H., Freeman, H. E., Lipsey, M. W ( 2003). Evaluation: A systematic approach. (7th Edn). London: Sage Publications.

Rowe, N. F. (2014). Sporting capital: a theoretical and empirical analysis of sport participation determinants and its application to sports development policy and practice. International Journal of Sport Policy and Politics, 7(1), 43-61. doi: $10.1080 / 19406940.2014 .915228$ 
Schulenkorf, N. (2012). Sustainable community development through sport and events: A conceptual framework for Sport-for-Development projects. Sport Management Review, 15(1), 1-12. doi: http://dx.doi.org/10.1016/j.smr.2011.06.001

Sherry, E. (2016) 'The Community Street Soccer Program.' In Conrad, D. and White, A. (eds.) Sports-Based Health Interventions., New York: Springer, pp. 181-188.

Sisjord, M. K. (2015). Assessing the sociology of sport: On lifestyle sport and gender. International Review for the Sociology of Sport, 50(4-5), 596-600. doi: $10.1177 / 1012690214552432$

Spinney, J. (2010). Performing Resistance? Re-Reading Practices of Urban Cycling on London's South Bank. Environment and Planning A, 42(12), 2914-2937. doi: $10.1068 / \mathrm{a} 43149$

Taks, M., Chalip, L., and Green, B. C. (2015) 'Impacts and strategic outcomes from nonmega sport events for local communities.' European Sport Management Quarterly, 15(1), pp. 1-6.

Taks, M., Green, B. C., Misener, L., \& Chalip, L. (2014). Evaluating sport development outcomes: the case of a medium-sized international sport event. European Sport Management Quarterly, 14(3), 213-237. doi: 10.1080/16184742.2014.882370

Weed, M. (2016). Should we privilege sport for health? The comparative effectiveness of UK Government investment in sport as a public health intervention, International Journal of Sport Policy and Politics, 8 (4) 559-576

Weed, M., Coren, E., Fiore, J., Wellard, I., Chatziefstathiou, D., Mansfield, L., et al. (2015). The Olympic Games and raising sport participation: a systematic review of evidence and an interrogation of policy for a demonstration effect. European Sport Management Quarterly, 15(2), 195-226. doi: 10.1080/16184742.2014.998695

Weed, M., Coren, E., Fiore, J., Wellard, I., Mansfield, L., Chatziefstathiou, D. and Dowse, S., (2012). Developing a physical activity legacy from the London 2012 Olympic and Paralympic Games: a policy-led systematic review. Perspectives in public health, 132(2), pp.75-80.

Widdop, P., King, N., Parnell, D., Cutts, D. and Millward, P. (2018). Austerity, policy and sport participation in England. International Journal of Sport Policy and Politics, 10 (1), 7-24. doi: 10.1080/19406940.2017.1348964

Yin, R. K. (2009) Case study research: design and methods. $4^{\text {th }}$ Edn. London: Sage publications. 

